\title{
A Vibration Energy Harvesting Sensor Platform for Increased Industrial Efficiency
}

\author{
Todd A. Anderson, ${ }^{*}$ Daniel W. Sexton \\ GE Global Research, One Research Circle, Niskayuna, NY, USA 12309
}

\begin{abstract}
A model for piezoelectric vibration energy harvesting with a piezoelectric cantilever beam is presented. The model incorporates expressions for variable geometry, tip mass, and material constants, and allows the parameterized determination of the voltage and power produced over a purely resistive load. The model is of a lumped-element form, with the base excitation acceleration and voltage representing the effort variables, and the tip velocity and electrical current representing the flow variables. Subsequent to the model's derivation, experimental results are presented and demonstrate the accuracy of the model. As peak power output for existing vibration configurations is typically of interest, several simple optimization studies are then performed on a simple generator configuration to demonstrate the effects of several of the driving geometric and material parameters.
\end{abstract}

\section{Introduction}

Asset health monitoring is attractive for increasing reliability, performance and efficiency. Particularly in industrial environments, condition monitoring of rotating machinery such as electric motors, vacuum pumps and compressors offers a significant opportunity for overall energy savings. By using data published in Department of Energy (DOE) sponsored publications it is estimated that through increased electric motor monitoring and subsequent improved maintenance and proper sizing, an opportunity of 122 trillion BTU of energy exists to be saved by the year 2020. To address this need, the Department of Energy has recently sponsored several research programs to develop technology to support industrial wireless condition monitoring.

Aspects of the "Distributed Wireless Multi-Sensor Technologies" program at the General Electric Global Research Center will be described in this paper. This research program focuses on the development of a sensor platform for multi-measurand data acquisition and an accompanying industrial wireless protocol. An overview of the program is presented in Figure 1. To achieve the battery lifetime and life cycle cost requirements for the sensor platform, harvesting ambient energy from the sensor's environment was investigated.

Technology to extract energy from the local environment for some useful purpose is generally termed "energy harvesting" or "power scavenging." With this technology, the use of a large storage battery to power health-monitoring sensors becomes unnecessary and a theoretically infinite life is achieved, provided there is adequate energy to harvest. Perhaps even more significant is the elimination of wiring requirements, which can often require larger investment than the sensors themselves. Energy for the wireless sensors can be extracted from many forms such as UV, thermal, pressure, and vibration. Roundy et al. present thorough reviews and a comparison of energy sources. ${ }^{1,2}$

Particularly for the rotating machinery being targeted for this sensor system, vibration energy is an attractive source of electrical energy. The mechanism by which this vibration energy is converted to electrical power can also be one of several schemes such as: electromagnetic, piezoelectric, and electrostatic. Although the best type of vibration energy harvesting scheme often depends on the particular sensor's environment, in terms of the power density, piezoelectric vibration energy harvesting performs well compared to the other technologies and was selected for investigation. ${ }^{1}$ Williams et al. present a useful simple model to predict the maximum output of vibration-powered devices. ${ }^{3}$

Piezoelectric vibration energy harvesting describes a scheme in which a piezoelectric element is harmonically strained to generate an $\mathrm{AC}$ voltage potential across the element's electrodes. Because the usual goal is to maximize the

*E-mail: andersot@research.ge.com; Phone: 518-387-5969; Fax: 518-387-5459; www.research.ge.com

Smart Structures and Materials 2006: Sensors and Smart Structures Technologies for Civil,

Mechanical, and Aerospace Systems, edited by Masayoshi Tomizuka, et al., Proc. of SPIE Vol. 6174, 61741Y, (2006) - 0277-786X/06/\$15 - doi: 10.1117/12.659586 
electrical power from generally low vibration levels, structural resonance is used to amplify the average strains within the piezoelectric element, and thereby increasing the power output. Using the structure's resonance is of course somewhat limiting however, because the device is only designed to resonate at one particular driving frequency. Despite this limitation, the host structures targeted for condition health monitoring (e.g., electric motors, pumps, compressors) are typified by a constant vibration spectrum and do not pose a significant challenge for vibration energy harvesting. Sodano et al. present a thorough review of piezoelectric vibration energy harvesting. ${ }^{4}$

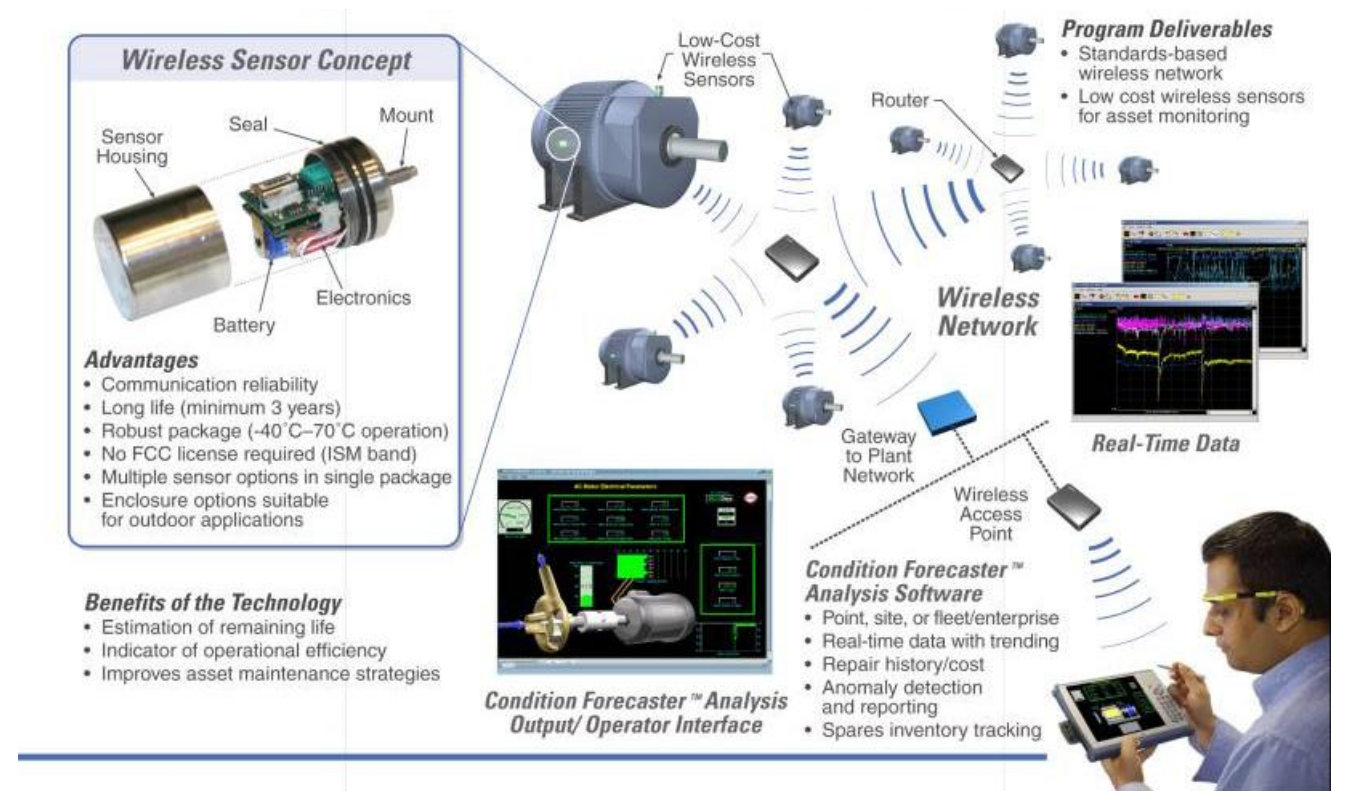

Figure 1. Description of the research program.

The piezoelectric energy-harvesting demonstration device designed for the sensor package consisted of a 3-layer piezoelectric composite cantilever beam. The top and bottom layers of the beam were piezoelectric PZT-5A and the center layer is a reinforcing metallic shim. The beam is tuned to resonate at one of the critical modes of an electric motor; these modes are a function of the driving AC line frequency and number of poles of the electric motor. Tuning was accomplished through the addition of an adjustable tip mass to the end of the cantilever beam. The electric charge and corresponding current are accessed through wires bonded to the electrode surfaces of the piezoelectric layers. The power conditioning circuitry used to convert the alternating high voltage signal into a stable DC power source and novel battery augmentation power control circuit was also designed and integrated into the experimental device.

As maximum power output as a function of mass and volume are of primary interest, a piezoelectric generator model has been developed and will be presented in this article. The model is in a lumped-element form, with the base excitation acceleration and voltage representing the effort variables, and the tip velocity and electrical current representing the flow variables. As it is a lumped element model, it is only appropriate for conditions where the device is being driven primarily near or below the first vibration mode, which is the case for these motor applications. Optimization studies are then performed on a simple generator configuration to demonstrate the effects of several of the driving geometric and material parameters. Experimental results are also presented to demonstrate the accuracy of the model.

Finally, the results of preliminary tests using the vibration energy harvesting device and accompanying power conditioning circuit on industrial electric motors will be presented. In these tests it was found that approximately 200 microwatts of power could be achieved on a typical industrial 20-hp two-pole electric motor.

\section{Device Design}

Because of its lower resonant frequency and higher average strains, the cantilever beam configuration is chosen as the piezoelectric generator. The geometry of the device modeled is presented in Figure 1. The bimorph is actually a 3- 
layer beam that includes a metallic center shim that is commonly incorporated to increase the structural durability of the ceramic piezoelectric element. The width of the piezo, shim and electrode layers is represented by the variable, $w$.

An electrode of negligible thickness begins at the cantilever root and terminates at length $l_{e}$, which for the remainder of this study will be defined to be equal to $l-l_{m}$. The tip mass is included to decrease the resonant frequency and is modeled to be symmetrically located at the end of the beam.

Series configuration bimorphs (poling directions in each of the piezoelectric layers point in opposing directions) generate twice the voltage compared to parallel configuration bimorph beams (poling directions point in the same direction) and are thus attractive for generating higher voltages necessary to forward bias rectifying circuits, despite the equal power output of parallel and series configurations. Accordingly, the model developed in this study will focus on the series configuration piezoelectric bimorph.

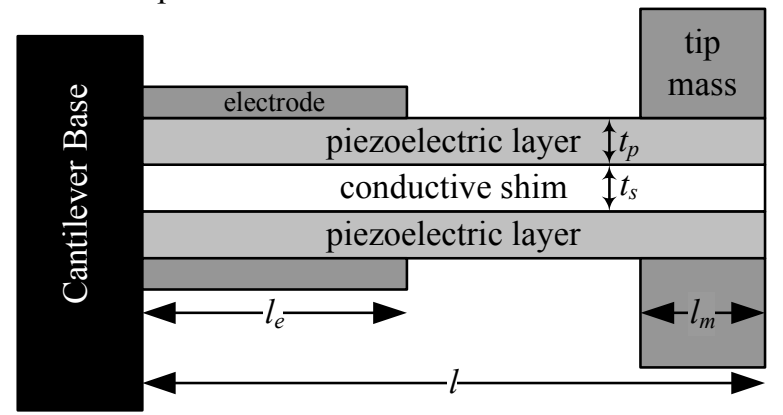

Figure 2. Schematic of the generator and variable definition.

\section{Analysis Model}

A first step toward the development of a model to predict power output of the piezoelectric generator device is to determine the coupling relationship between the tip displacement of the beam and the voltage applied to the beam. For the configuration of interest, and with the variables defined in Figure 2, this relationship for the displacement-voltage coupling coefficient is determined to be

$$
d_{S}=\frac{3 d l\left(l-l_{m}\right)\left(t_{p}+t_{s}\right) Y_{p}}{8 t_{p}^{3} Y_{p}+12 t_{p}^{2} t_{s} Y_{p}+6 t_{p} t_{s}^{2} Y_{p}+t_{s}^{3} Y_{s}}
$$

where $d$ is the piezoelectric strain coefficient, and $Y_{p}$ and $Y_{s}$ are the Young's moduli of the piezoelectric and shim materials, respectively. Thus the free tip displacement, $\delta$, is related to the applied voltage by the relationship, $\delta=d_{s} V$. If the beam is simplified to be a 2-layer bimorph $\left(t=2 t_{p}, t_{s}=0\right)$ and to have a point mass at $x=l\left(l_{m}=0\right)$, the relationship simplifies to the well-known relationship

presented in Smits et al. ${ }^{5}$

$$
d_{S}=\frac{3 d l^{2}}{2 t^{2}}
$$

The quasi-static behavior of the electromechanically coupled 3-layer beam can then be determined using an equation similar to the strain-charge piezoelectric constitutive relations and written as

$$
\left\{\begin{array}{l}
\delta \\
q
\end{array}\right\}=\left[\begin{array}{ll}
C_{S} & d_{S} \\
d_{S} & C_{F}
\end{array}\right]\left\{\begin{array}{l}
F \\
V
\end{array}\right\}
$$

with $q$ representing the charge, $C_{s}$ the short-circuit mechanical compliance, $F$ the applied force at the centroid of the tip mass, and $C_{F}$, the free capacitance of the device. The short-circuit $(V=0)$ tip compliance, $C_{S}$, is defined as the tip displacement per unit of force applied at the tip of the cantilever beam. Using the composite second area moment of the 3 -layer beam and the deflection of the beam at the center of the mass $\left(x=l-l_{m} / 2\right)$, the tip compliance can be determined to equal 


$$
C_{S}=\frac{\left(2 l-l_{m}\right)^{3}}{4 t_{p}\left(4 t_{p}^{2}+6 t_{p} t_{s}+3 t_{s}^{2}\right) w Y_{p}+2 t_{s}^{3} w Y_{s}}
$$

If the beam being analyzed reduces to a two-layer bimorph with no piezoelectric shim $\left(t=2 t_{p} ; t_{s}=0\right)$, and the mass is assumed to be a point mass at the tip of the beam $\left(l_{m}=0\right)$, the compliance simplifies to,

$$
C_{S}=\frac{4 l^{3}}{w t_{p}^{3} Y_{p}} \text {. }
$$

Lastly, the free-stress capacitance of the series configuration device, $C_{F}$, is defined as

$$
C_{F}=\frac{\varepsilon_{T} w l_{e}}{2 t_{p}}
$$

where $\varepsilon_{T}$ is the free permittivity of the piezoelectric material. The thickness of the shim material is not included in the distance between the electrodes as it is a metallic conductor.

With the strain-charge form of the equations fully defined, the quasi-static behavior of electromechanically coupled beam is complete. However, the low-frequency harmonic response of the system can also be obtained by differentiating with respect to time, resulting in

$$
\left\{\begin{array}{l}
\dot{\delta} \\
i
\end{array}\right\}=\left[\begin{array}{ll}
j \omega C_{S} & j \omega d_{S} \\
j \omega d_{S} & j \omega C_{F}
\end{array}\right]\left\{\begin{array}{l}
F \\
V
\end{array}\right\} ; j=\sqrt{-1}
$$

where $\dot{\delta}$ is the tip velocity, $i$ is the current, and $\omega$ is the angular velocity. For later use in the model, the ratio of applied force to the voltage output is also necessary. To determine the this relationship, the strain-charge form of the equations can be rearranged to form the stress-charge form of the equations,

$$
\left\{\begin{array}{c}
F \\
i
\end{array}\right\}=\left[\begin{array}{cc}
\frac{1}{j \omega C_{S}} & -\frac{d_{S}}{C_{S}} \\
\frac{d_{S}}{C_{S}} & j \omega C_{B}
\end{array}\right]\left\{\begin{array}{l}
\dot{\delta} \\
V
\end{array}\right\} .
$$

Thus, the coupling relationship between an applied voltage and its generated blocked force is equal to

$$
\phi=-\frac{d_{S}}{C_{S}} .
$$

In the process of deriving the stress-charge form of the equations, an additional relationship for the blocked capacitance is defined as

where

$$
C_{B}=C_{F}\left(1-k^{2}\right)
$$

$$
k^{2}=\frac{d_{S}^{2}}{C_{S} C_{F}} .
$$

It is important to note that this $k$ is a system electromechanical coupling coefficient and not the usual material electromechanical coupling coefficient.

The strain-charge and stress-charge equations presented in Equations 7 and 8 are only valid for very low frequency harmonic, or quasi-static excitation. For the velocities and frequencies of interest, a more sophisticated model that includes mechanical inertia and damping is necessary. Using an impedance analogy, the velocity of the tip and electrical current, and the applied force and voltage, are equivalent quantities and thus an equivalent electrical circuit model can be used to describe the coupled system. With this analogy, the circuit resistance is equivalent to the mechanical damping parameter, $D$, and the inductance is equal to the tip mass, $m$. Using these lumped element parameters, it is then possible to construct an appropriate 2-port electrical circuit model. The circuit, displayed in Figure 3, includes the transformer ratio, $\phi$, to convert the effort variable force, to the effort variable voltage, and vice 
versa. The resistance on the electrical side of the model, $R$, represents the resistive load that will be dissipating the power generated by the piezoelectric device.

To this point, the model has assumed that an applied force would be driving the beam at the centroid of the tip mass. However for the purpose of energy harvesting, the beam structure will be driven by base excitation. Therefore, the applied force will equal the tip mass multiplied by the base excitation acceleration, $F=m A$.

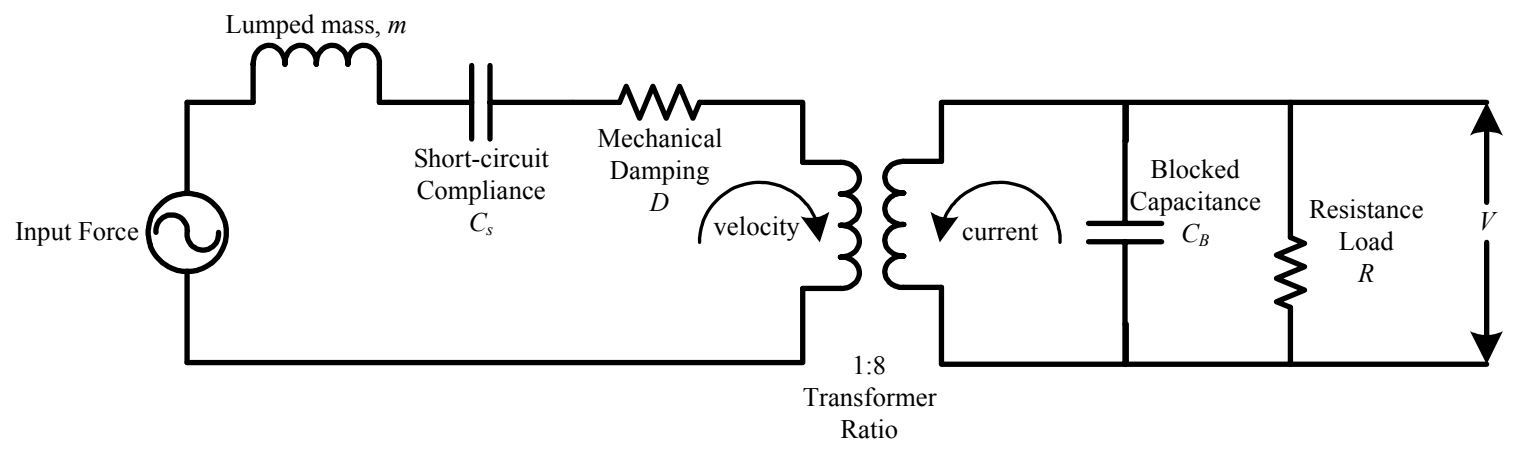

Figure 3. Schematic if the lumped element circuit model.

With all of the lumped circuit parameters defined, the relationship for the voltage, or power can be derived using electric circuit theory. The impedance of the three series elements on the mechanical side of the model can be represented by

$$
Z_{S}=m\left(2 \omega_{n} \zeta+j \omega\left(1-\frac{\omega_{n}^{2}}{\omega^{2}}\right)\right)
$$

with $\zeta$ equal to the damping ratio and $\omega_{n}$ equal to the short-circuit natural frequency of the vibrating beam,

Similarly, the impedance of the electrical side is

$$
\omega_{n}^{2}=\frac{1}{C_{S} m}
$$

$$
Z_{p}=\frac{j R}{j-C_{B} \omega R}
$$

and when brought over to the mechanical side is simply multiplied by the transformer ratio squared,

$$
Z_{p}^{\prime}=\frac{\phi^{2} j R}{j-C_{B} \omega R}
$$

With the two impedances and the applied force, one can solve for the voltage across the transformer, or by using the transformer ratio, the voltage across the resistive load on the right hand side of the model. This results in

$$
V=\frac{A m \omega \phi R}{m\left(C_{B} \omega R-j\right)\left(2 j \omega_{n} \omega \zeta-\omega_{n}^{2}-\omega^{2}\right)-\omega \phi^{2} R}
$$

for the voltage over the resistive load. The power dissipated over the real resistance is determined by $V^{2} / R$, or

$$
P=\frac{(A m \omega \phi)^{2} R}{\left(\omega \phi^{2} R-m\left(C_{B} \omega R-j\right)\left(\omega^{2}-\omega_{n}^{2}-2 j \omega_{n} \omega \zeta\right)\right)^{2}} .
$$

\section{Experimental Method}

To evaluate the accuracy of the voltage and power predictions by the lumped-element piezoelectric generator model described in the previous section, several experiments are performed. The configuration of the beam to be tested 
matches that presented in Figure 1, with two piezoelectric layers bonded to a brass shim. The beam evaluated in this study has a free length, $l$, of $23.1 \mathrm{~mm}$. The thickness of the piezoelectric layers and the brass shim are $1.1 \mathrm{~mm}$ and 1.1 $\mathrm{mm}$, respectively. The width of all elements in the assembly is $6.35 \mathrm{~mm}$.

The piezoelectric 3-layer beam was purchased from Piezo Systems Co. (MA) and is model T215-A4-203X. The piezoelectric material used in this bimorph is PZT-5A and both layers are poled for series connections (Xconfiguration). The Young's modulus of the PZT is $66 \times 10^{9} \mathrm{~Pa}$ and the modulus of the brass shim layer is $117 \times 10^{9} \mathrm{~Pa}$. The relative dielectric constant given by the manufacturer for the PZT layers is 1800 .

The tip mass is included to reduce the first mode resonance to $60 \mathrm{~Hz}$. To accomplish this, brass masses $4.5 \mathrm{~mm}$ long are bonded to the top and bottom of the beam using a small amount of epoxy adhesive. The widths of the masses are equal to the width of the piezoelectric element. The total mass of the two masses, including the additional adhesive, is 4.8 gm.

The cantilever beam is subjected to base excitation through the use of an electromechanical shaker (Bruel \& Kjaer 4809). The shaker is driven with a sine wave generator provided by an Agilent Dynamic Signal Analyzer (HP35670A) and amplified using a broadband Carver PM-300 amplifier. For all experiments and comparisons, the base excitation acceleration used for these studies is $195 \mathrm{mG}$. The voltage of the excited cantilever beam is obtained using the Agilent Dynamic Signal Analzyer that is connected across a resistor of $400 \mathrm{kohm}$.

\section{Results and Discussion}

\subsection{Model Verification}

The voltage over a $400 \mathrm{kOhm}$ resistor generated by the vibrating cantilever beam and the values of the expressions developed in Section 3 are first examined. Figure 5 presents the voltages predicted by the model, for two different levels of mechanical damping, as a function of the excitation frequency. As can be seen the mechanical damping that best captures the experimental behavior would be approximately $2 \%$, which is a common value for typical structural systems. The analytical results over predict the actual resonant frequency by approximately 2 Hertz. Considering the assumptions included in a lumped element model however, good agreement is found between the experiment and analytical predictions.

\subsection{Electric Motor Demonstration Results}

A test platform consisting of the device described in Section 4 was constructed. The device was constructed with an adaptable mount to allow attachment to various electric motors. For demonstration purposes the device was attached to a 20-horsepower 2-pole three-phase AC induction motor. Horizontal vibration was measured to be approximately $80 \mathrm{mG}$ at $60 \mathrm{~Hz}$. The voltage generated was measured over a purely resistive load and then related to the power output of the device. The peak output power of the unoptimized device was $195 \mu \mathrm{W}$ at approximately $450 \mathrm{k} \Omega$.

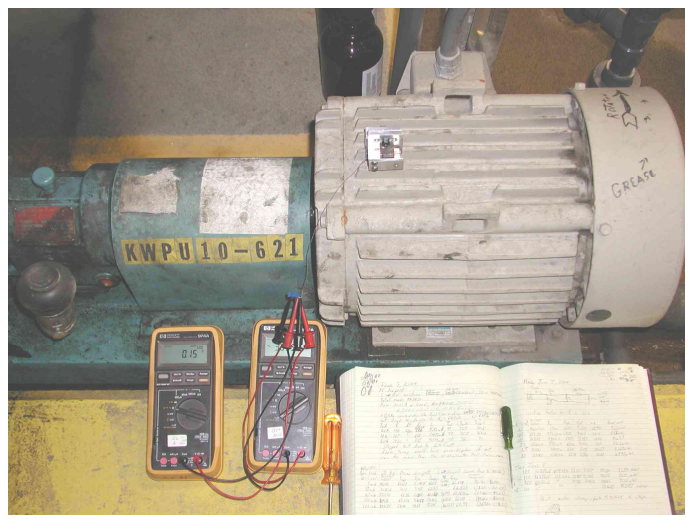

Figure 4. Picture of the energy harvesting test platform attached to an $\mathrm{AC}$ induction motor. 


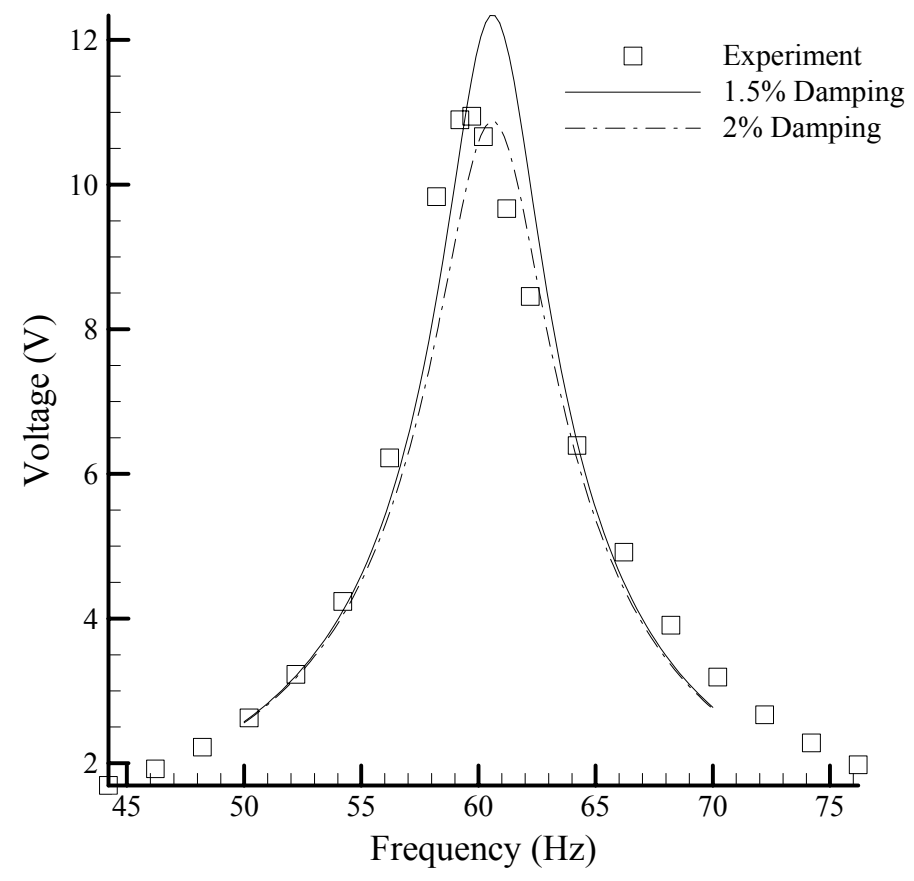

Figure 5. The variation of voltage as a function of the driving frequency for the experiment and for modeled cases including $1.5 \%$ and $2 \%$ damping.

\subsection{System Optimization}

The previous experiments were performed on device with a suboptimum set of material and geometric parameters. As it would be beneficial to maximize the power of any device, it would follow that it would be advantageous to perform an optimization using the lumped element model derived in Section 3. The results of such an optimization will now be presented to examine the effects of the various system parameters.

Although there are many variables that define the lumped element system, this study will be limited to examine the geometrical and mass parameters. In many structural health-monitoring applications, the base excitation frequency is a known, constant frequency and the energy-harvesting device must be designed to resonant at that frequency. Therefore these optimization studies will constrain the range of resonance to $59-60 \mathrm{~Hz}$, which would be appropriate for structures being driven for example by a 2-pole AC motor.

Similarly, the device being powered by the energy-harvesting device will most likely have a defined resistance load. Although it is possible to boost that resistance, it is generally not advantageous to do so, and reducing the resistance load is not easily accomplished without wasting power on parallel load paths. Therefore, the optimization studies will be performed for several fixed values of resistant loads, from 100 to $500 \mathrm{kOhm}$, in increments of $100 \mathrm{kOhm}$.

The optimization design variables chosen for these analyses are mass, which is constrained to be less than 10 grams, length, which is defined to be less than $25 \mathrm{~mm}$, and width, which is constrained to be less than $10 \mathrm{~mm}$. The objective function to be minimized, (1-2000P), performs well and converges easily. All material properties and other defining geometry parameters remain equal to those defined in Section 4.

The results of the five different optimization analyses are presented in Table 1. As can be observed, the mass drives the power output of the devices and in all cases, the optimum mass is equal to the upper bound for these analyses. The 
remaining design variables, length and width, vary as one would expect considering the fixed natural frequency of 60 $\mathrm{Hz}$.

Table 1. Results of the five optimization sets.

\begin{tabular}{lccccc} 
& \multicolumn{5}{c}{ Optimization Set } \\
& 1 & 2 & 3 & 4 & 5 \\
\hline target resistance $(\mathrm{kOhm})$ & 100 & 200 & 300 & 400 & 500 \\
length $(\mathrm{mm})$ & 21.2 & 19.1 & 17.4 & 16.3 & 15.5 \\
mass $(\mathrm{gm})$ & 10 & 10 & 10 & 10 & 10 \\
width $(\mathrm{mm})$ & 10 & 7 & 5.1 & 4.1 & 3.4 \\
power $(\mathrm{uW})$ & 726.2 & 734.6 & 734 & 733.5 & 733.2 \\
width*length*resistance & $2.12 \mathrm{E}+07$ & $2.67 \mathrm{E}+07$ & $2.66 \mathrm{E}+07$ & $2.67 \mathrm{E}+07$ & $2.63 \mathrm{E}+07$
\end{tabular}

The power output as a function of the resistance load for the five-optimization sets is presented in Figure 6. As the figure shows, the curves are generally sharper for the devices that are optimized for lower resistance loads. This is of importance for devices that will have varying loads (e.g., when a wireless sensor goes from monitor to transmit) as the power generated will vary significantly. It would follow from these results that it would be advantageous to optimize for higher resistance loads as the decline when the resistance deviates from the optimum value is significantly less.

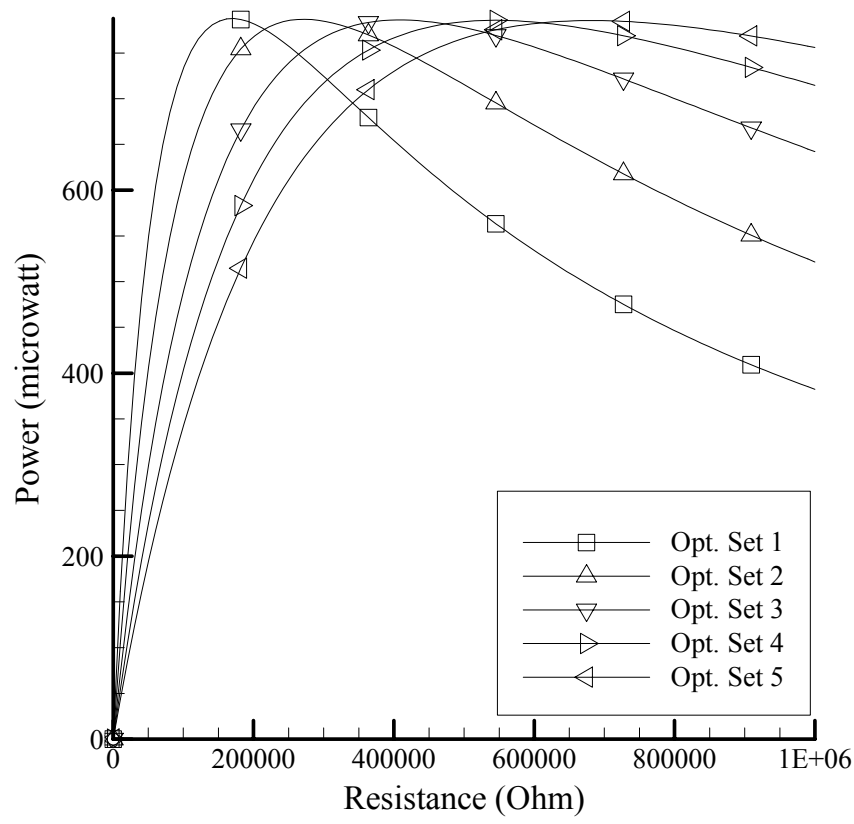

Figure 6. The variation of power as a function of resistance for the optimized cases.

It is also of interest that the peak power output of any of the optimized devices appears to approach a constant value as can be observed in Figure 6. The only case that deviates slightly is Case 1, but that is most likely due to the width variable hitting its upper bound. This is result is really just an artifact of the optimization parameters and the constraint on the constant natural frequency and will be explained by the following: the piezoelectric energy harvester will produce peak power when the impedance of the load, including the piezoelectric device, is equal to the conjugate of the mechanical impedance (Equation 12). Because every system will produce optimum power when the driving frequency equals approximately the mechanical natural frequency, the impedance of the mechanical elements at resonance is simply equal to the mechanical resistance. Using the equations for these expressions derived in Section 3, one can 
determine that the peak power is obtained when the resistance of the mechanical side equals the resistive load. The implication of this is that $w \times l \times R$ is approximately equal to a constant value. This conclusion proves accurate for the five different optimization sets as is presented in the last row of Table 1. This conclusion will hold true only for cases where the driving frequency is kept constant and where only the width, length and mass are varied. For other designs, the maximum power will of course vary.

\section{Summary}

A model for piezoelectric vibration energy harvesting with a piezoelectric cantilever beam has been presented. The model incorporates expressions for variable geometry, tip mass, and material constants, and allows the parameterized determination of the voltage and power produced over a purely resistive load. Subsequent to the model's derivation, experimental results were presented and demonstrated the accuracy of the model. The effects on the system of several of the driving geometric and material parameters were illustrated through a brief optimization study on maximum power with respect to a given resistive electric load.

\section{Acknowledgment}

This work was partially supported by the Department of Energy under contract DE-FC36-04GO14001.

\section{REFERENCES}

${ }^{1}$. S Roundy, PK Wright and JM Rabaey, Energy Scavenging for Wireless Sensor Networks: with Special Focus on Vibrations, Kluwer Academic Publishers, Boston, 2003.

2. S Roundy and PK Wright, "A Piezoelectric Vibration Based Generator for Wireless Electronics”, Smart Materials and Structures, 13:1131-1142, 2004.

3. CB Williams, RC Woods and RB Yates, "Feasibility Study of a Vibration Powered Micro-Electric Generator", IEEE Colloquium on Compact Power Sources, 7:1-3, 1996.

4. Sodano, H.A., Inman, D.J. and Park, G., "A Review of Power Harvesting from Vibration using Piezoelectric Materials," Shock and Vibration Digest, 36(3):197-205, 2004.

5 . JG Smits, SI Dalke and TK Cooney, "The Constituent Equations Of Piezoelectric Bimorphs," Sensors and Actuators A, 28:41-61, 1991. 